\title{
Weiterer Beitrag zur Wirkung der Faradisation der quergestreiften Muskulatur bei Krampfkranken.
}

\author{
Von \\ Priv.-Doz. Dr. Heinrich Fiseher und Dr. Erika Thaer. \\ (Aus der Klinik für psychische und nervöse Krankheiten, Gießen.)
}

(Eingegangen am 23. Oktober 1921.)

Die im folgenden mitgeteilten Untersuchungen bilden die Fortsetzung einer in der gleichen Zeitschrift erschienenen Arbeit von FischerSchlund ${ }^{1}$ ). Es wurde bei gleicher Versuchsanordnung weiterhin das Verhalten der Blutgerinnung, der Zahl der roten Blutkörperchen und des Cholesteringehaltes des Blutes untersucht. Zur Bestimmung der Blutgerinnungszeit benutzten wir den $B$ ürkerschen Blutgerinnungsapparat und zur Zählung der roten Blutkörperchen die Bürkersche Zählkammer. Den Cholesteringehalt bestimmten wir nach der colorimetrischen Methode von A u tenrieth und Funk. Alle Untersuchungen wurden zur gleichen Tageszeit - nachmittags zwischen 5 und $61 / 2 \mathrm{Uhr}-$ und unter den gleichen Versuchsbedingungen ausgeführt; diese waren Bettruhe bis zum Versuch, gleiche Ernährung, bequeme Ruhelage und gleicher Untersuchungsraum bei gleicher Temperatur. In der Technik der Untersuchungen über die Blutgerinnungszeit haben wir uns ganz an die Vorschrift Bürkers²) gehalten. Wir verweisen ebenso wie zur Kritik der von anderen Autoren benutzten Methoden auf seine dies. bezügliche Veröffentlichung. Im Interesse der Kürze der Arbeit soll auch nicht auf die Theorie des Blutgerinnungsprozesses eingegangen werden. Betont sei nur, daß nach Bürker die Blutgerinnungszeiten bei verschiedenen gesunden Individuen an verschiedenen Tagen bei annähernd gleicher Temperatur und bei annähernd gleicher Tageszeit untersucht nahezu gleich sind, und zwar wird die normale Blutgerinnungszeit von Bürker auf 5 Minuten angegeben.

Bestimmungen der Blutgerinnungszeit sind bei verschiedenen psychischen Krankheitsbildern untersucht worden. Ha u pt ma n $n^{3}$ ) unter-

1) Diese Zeitschr. 72. 1921. Zur Wirkung der Faradisation der quergestreiften Muskulatur bei Krampfkranken und bei Gesunden.

2) Bürker, Vereinfachte Methode zur Bestimmung der Blutgerinnungszeit. Arch. f. d. ges. Physiol. 149.

3) Diese Zeitschr. 29. 1915. 
suchte außer bei einer Reihe anderer Krankheiten die Blutgerinnungszeit bei Epilepsie. Er fand hierbei die Gerinnungszeit oberhalb der normalen Linie, also verzögert. E. Sch neider ${ }^{1}$ ) untersuchte nach der Methode von Fingerhut und Wintz Fälle von Katatonie, Hysterie, Epilepsie und Paralyse. Nach seinen Untersuchungen weisen diese Krankheitsvorgänge - besonders im Durchschnittswert - bis auf minimale, fast noch innerhalb der Fehlergrenzen liegende Abweichungen die gleiche Blutgerinnungszeit auf. De Crinis ${ }^{2}$ ) fand bei Epilepsie ,,im allgemeinen die Blutgerinnung häufig beträchtlich verzögert" „,Vor Anfällen läßt sich jedesmal eine Verzögerung der Blutgerinnung beobachten, die nach dem Anfall sofort abnimmt und bis zur Norm zurückkehren kann." Der Unterschied in der Gerinnungszeit vor und nach dem Anfall soll immer in die Augen springend sein.

Über die Wirkung aktiver Muskelarbeit auf das Verhalten der Blutgerinnungszeit liegen anscheinend noch keine Untersuchungen vor. U.E. sind vergleichende Untersuchungen über das Verhalten der Blutgerinnungszeit bei Muskelarbeit sehr wichtig, da nur so festzuztellen ist, ob die bei Krämpfen erhobenen Resultate der Blutgerinnungszeit nicht lediglich Folge der im Krampfe geleisteten Muskelarbeit sind, wie auch andere beim Krampf beobachtete Stoffwechselvorgänge. Sehr wichtig scheint uns ferner, daß Verzögerung der Blutgerinnung auch nach Adrenalininjektion festgestellt ist. Die Faradisation der quergestreiften Muskulatur ist als passive Muskelarbeit anzusehen. Wir haben unsere Untersuchungen auf das Verhalten der Blutgerinnungszeit während der Faradisation insbesondere auch deshalb ausgedehnt, um an Hand der schon bei Epilepsie vorliegenden Untersuchungsbefunde vergleichen zu können, ob die Ergebnisse etwas für den Krampfanfall Spezifisches darstellen.

I. S. 32 jähriger Arbeiter. Diagnose: genuine Epilepsie.

\begin{tabular}{|c|c|c|c|}
\hline $\mathrm{I}$ & $\begin{array}{c}\text { Blutgerinnungs- } \\
\text { zeit }\end{array}$ & $\begin{array}{l}\text { Erythrocyten } \\
\text { im ccm Blut }\end{array}$ & Cholesterin \\
\hline $5^{\mathrm{h}} 10^{\prime}$ & $6 \frac{1}{2}$ Min. & 4,18 Mill. & $0,255 \%$ \\
\hline \multicolumn{4}{|c|}{$5^{\mathrm{h}} 30^{\prime}-6^{\mathrm{h}} 05^{\prime}$ Faradisation } \\
\hline $\begin{array}{l}5^{\text {h } 40^{\prime}} \\
5^{\text {h }} 50^{\prime} \\
6^{\text {h }}\end{array}$ & $\begin{array}{ll}6^{1 / 4} & \text { Min. } \\
4^{1 / 4} & n \\
3^{3} / 4 & n\end{array}$ & $\begin{array}{l}4,45 \text { Mill. } \\
4,42 \quad " \\
4,12 \quad "\end{array}$ & $\begin{array}{c}\text { - } \\
0,243 \%\end{array}$ \\
\hline $6^{\mathrm{h}} 20^{\prime}$ & $5^{1} / 4$ Min. & 4,10 Mill. & 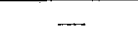 \\
\hline
\end{tabular}

Bei der Faradisation ist $\mathrm{S}$. wie auch sonst meistens etwas ängstlich. Die Muskulatur zeigt Reizerscheinungen in Form von Tremor und Wogen.

1) Monatsschr. f. Psychiatr. u. Neurol. 45. 1919.

2) Die Beteiligung der humoralen Lebensvorgänge des menschlichen Organismus im epileptischen Anfall. 1920. 
Die Pupillen werden fast maximal weit und different (r. $>1$.). Gegen Schluß klagt Pat. über Klingen im linken Ohr.

Das wesentliche Ergebnis dieses Versuches sind bemerkenswerte Veränderungen in der Blutgerinnungszeit. Zunächst ist hervorzuheben, da $\beta$ die Blutgerinnung im Vorversuch verzögert ist. Sie beträgt 6,5 Min. gegenüber der normalen Zeit von 5 Min. Während der Faradisation zeigt sich gleichmäßig zunehmend eine Verkürzung der Blutgerinnungszeit bis auf 3,75 Min., also um $42 \%$. Weiter ist zu betonen, daß diese Verkürzung mit Aufhören der Faradisation rasch wieder abnimmt und die Blutgerinnungszeit nach 15 Min. wieder auf 5,25 Min. verzögert ist.

Ferner sei auf den ungewöhnlich hohen Cholesteringehalt des Blutes hingewiesen. Nach Step p schwanken die normalen Zahlen des Cholesteringehaltes des Blutes zwischen 0,13 und 0,17. Der hohe Cholesteringehalt fand sich in diesem Falle bei zweimaliger Untersuchung, und zwar ohne daß Pat. weder tagelang vorher noch tagelang nachher einen Anfall hatte. Danach scheint ein gesetzmäßiges Verhalten zwischen der Höhe des Cholesteringehaltes im Blut und dem Auftreten eines Anfalles zweifelhaft. Weiter ergaben die Untersuchungen von De Crinis, daß die Kurve des Cholesteringehaltes bei Epilepsie eine bedeutende Abweichung von der Norm zeigt und die Grenzen der normalen Werte häufig um ein beträchtliches überschritten werden. Nach seinen Untersuchungen soll der Cholesteringehalt vor dem Anfall regelmäßig ein wenig ansteigen, mit dem Anfall sein Maximum erreichen und nach dem Anfall absinken, um zu normalen Werten zurückzukehren.

II. St., 20 jähriger Arbeiter. Diagnose: genuine Epilepsie.

\begin{tabular}{c|c|c}
\hline II & $\begin{array}{c}\text { Blutgerinnungs- } \\
\text { zeit }\end{array}$ & Cholesterin \\
\hline $5^{\mathrm{h}} 15^{\prime}$ & 7 Min. & $0,127 \%$ \\
\hline $5^{\mathrm{h}} 30^{\prime}-6^{\mathrm{h}} 05^{\prime}$ Faradisation \\
\hline $5^{\mathrm{h}} 40^{\prime}$ & 6 Min. & - \\
$5^{\mathrm{h}} 50^{\prime}$ & $4^{1} / 2$ & $0,127 \%$ \\
$6^{\mathrm{h}}$ & $4^{1} / 2$, & - \\
\hline $6^{\mathrm{h}} 25^{\prime}$ & $5^{1} / 2 \mathrm{Min}$. & $0,153 \%$
\end{tabular}

Während der Faradisation leichte motorische Reizerscheinungen und Pupillenerweiterung. Nach der Faradisation Müdigkeit und Schlaf.

Im Vorversuch deutliche Verzögerung der Blutgerinnungszeit (7 Min.), unter Einwirkung der Faradisation Verkürzung bis auf 4,5 Min., also um $36 \%$. Nach Schluß Wiedereinsetzen der Verzögerung.

Der Cholesteringehalt zeigte in diesem Fall von genuiner Epilepsie keine Erhöhung.

III. W., 20jähriger Mann. Ein Onkel mütterlicherseits leidet seit der Pubertätszeit an Ohnmachten..Pat. war vor der Pubertätszeit unauffällig, entwickelte 
sich normal. Seit dem 17. Lebensjahre anfallsartige Zuckungen in beiden Armen mit Beteiligung des Kopfes ohne Bewußtseinsverlust, besonders morgens früh beim Erwachen, niemals im Schlaf, vereinzelt auch nachmittags. Später kamen schwere Anfälle mit Bewußtseinsverlust, Krämpfen, Zungenbiß, Einnässen und terminalem Schlaf hinzu. Pat. fühlt jeden Anfall kommen. In der Klinik wurden nur myoklonische Erscheinungen beobachtet.

\begin{tabular}{|c|c|c|c|}
\hline III & $\begin{array}{c}\text { Blutgerinnungs- } \\
\text { zeit } \\
\end{array}$ & $\begin{array}{l}\text { Erythrocyten } \\
\text { im ccm Blut }\end{array}$ & Cholesterin \\
\hline $5^{\text {h }} 15^{\prime}$ & $4^{1} / 2$ Min. & 4,41 Mill. & $0,192 \%$ \\
\hline \multicolumn{4}{|c|}{$5^{\text {h }} 35^{\prime}-6^{\text {h }} 10^{\prime}$ Faradisation } \\
\hline $\begin{array}{l}5^{\text {h }} 40^{\prime} \\
5^{\text {h }} 50^{\prime} \\
6^{\text {h }}\end{array}$ & $\begin{array}{lc}3^{1} / 4 & \text { Min. } \\
4 & n \\
3^{3} / 4 & n\end{array}$ & $\begin{array}{lc}4,34 & \text { Mill. } \\
4,25 & \\
4,24 \quad,\end{array}$ & - \\
\hline $6^{\mathrm{h}} 16^{\prime}$ & $4^{1 / 4} \mathrm{Min}$. & 4,38 Mill. & $0,179 \%$ \\
\hline
\end{tabular}

Während der Faradisation Zittern bis zum Schluß. Nach 4 Min. Beginn der Pupillenerweiterung, die gegen Ende wieder abnimmt. Nach der Faradisation Müdigkeit und guter Schlaf. Die Blutgerinnungszeit hatte im Vorversuch normalen Wert, nahm unter dem Einfluß der Faradisation noch deutlich ab, aber nicht so stetig wie in den anderen Fällen, sondern unter leichtem Schwanken.

Der Cholesteringehalt des Blutes war im Vorversuch erhöht.

IV. E., 21 jähriger Mann. Diagnose: Katatonie mit elementaren Krämpfen.

\begin{tabular}{|c|c|c|}
\hline IV & $\begin{array}{c}\text { Blutgerinnungs- } \\
\text { zeit }\end{array}$ & $\begin{array}{l}\text { Erythrocyten } \\
\text { im ccm Blut }\end{array}$ \\
\hline $5^{\mathrm{h}} 15^{\prime}$ & $71 / 2$ Min. & 4,32 Mill. \\
\hline \multicolumn{3}{|c|}{$5^{\text {h }} 30^{\prime}-6^{\text {h }} 05^{\prime}$ Faradisation } \\
\hline $\begin{array}{l}5^{\text {h }} 45^{\prime} \\
5^{\text {h }} 55^{\prime} \\
6^{\text {h }} 05^{\prime}\end{array}$ & $\begin{array}{lc}5^{1} / 4 & \text { Min. } \\
4 & " \\
5 & " 1\end{array}$ & $\begin{array}{l}4,31 \text { Mill. } \\
4,16 " n \\
4,20 "\end{array}$ \\
\hline 0 & $5^{1 / 2}$ Min. & 4,17 Mill. \\
\hline
\end{tabular}

Während der Faradisation Tremor der gesamten Körpermuskulatur, geringe Erweiterung der Pupillen, die gleich nach Schluß wieder enger werden. Nach der Faradisation Müdigkeitsgefühl.

Auch in diesem Falle von Katatonie wurde eine bemerkenswerte Verzögerung der Blutgerinnungszeit auf 7,5 Min. gefunden. Wie bei den genuinen Epileptikern verkürzte sich die Blutgerinnungszeit während der Faradisation, und zwar um 40\%. Hervorzuheben ist hier, daß die Verzögerung der Blutgerinnung schon vor Schluß der Faradisation wieder einsetzte, um nach Aufhören weiter zuzunehmen.

V. Z., 24jähriger Arbeiter. Erlitt infolge eines leichteren Unfalles einen „Nervenschock“ (Angabe des Arztes). Nach dem erwähnten Unfall traten „Nervenanfälle" auf. Pat. kam ziemlich frisch in klinische Behandlung. Von Haus aus 
degenerative Persönlichkeit von nur mäßiger Begabung. Die Einwirkung eines schreckhaften Erlebnisses hatte bei ihm eine Reihe hysterischer Reaktionen (Zittererscheinungen, funktionelle Krämpfe, Sensibilitätsstörungen) ausgelöst, die unter der klinischen Behandlung verschwanden.

\begin{tabular}{|c|c|c|c|}
\hline $\mathrm{r}$ & $\begin{array}{c}\text { Blutgerinnungs- } \\
\text { zeit }\end{array}$ & $\begin{array}{l}\text { Erythrocyten } \\
\text { im ecm Blut }\end{array}$ & Cholesterin \\
\hline $5^{\mathrm{h}} 15^{\prime}$ & $5^{1 / 4}$ Min. & $5,30 \%$ & $0,176^{\circ} \%$ \\
\hline \multicolumn{4}{|c|}{$5^{\text {h }} 35^{\prime}-6^{\text {h }} 15^{\prime}$ Faradisation } \\
\hline $\begin{array}{l}5^{\text {h }} 4^{\prime} \\
5^{\text {h }} 53^{\prime} \\
6^{\text {h }} 11^{\prime}\end{array}$ & $\begin{array}{ll}4^{1} / 4 & \text { Min. } \\
3^{1 / 2} / 2 & " \\
2^{3} / 4 & ,\end{array}$ & $\begin{array}{l}4,98 \text { Mill. } \\
4,76 \quad " \\
4,92 \quad "\end{array}$ & $\begin{array}{c}-180 \% \\
-\end{array}$ \\
\hline $\begin{array}{l}6^{\text {h }} 24^{\prime} \\
6^{\text {h }} 35^{\prime} \\
6^{\text {h }} 45^{\prime}\end{array}$ & $\begin{array}{l}3 \quad \text { Min. } \\
4^{1 / 2} \quad "\end{array}$ & $\begin{array}{c}4,75 \text { Mill. } \\
5,08 \quad " \\
-\end{array}$ & $\begin{array}{c}- \\
- \\
0,130 \%\end{array}$ \\
\hline
\end{tabular}

Während der Faradisation Zittererscheinungen, die bis zum Schluß anhalten. Pupillen werden maximal weit, different $(1 .>$ r.), nach Schluß wieder enger, zunächst unter Beibehaltung der Differenz. Psychisch leicht euphorisch. Nach der Faradisation Müdigkeit und guter Schlaf.

Im Vorversuch zeigte die Blutgerinnungszeit normalen Wert. Sie nahm unter dem Einfluß der Faradisation gleichmäßig bis um $48 \%$ ab und nach der Faradisation ebenso gleichmäßig wieder zu. Der Cholesteringehalt lag im Vorversuch etwas über der oberen Grenze des Nor malen und hatte 30 Min. nach Schluß der Faradisation den unteren Normalwert erreicht.

Während der Faradisation auffallend starkes Ausfließen des Blutes. Ein ähnliches Verhalten im Ausfließen des Blutes wurde fast regelmäßig während der Faradisation beobachtet. Dies Ergebnis steht im anscheinenden Widerspruch zu der Tatsache, daß die Blutgerinnungszeit gleichzeitig eine zunehmende Verkürzung zeigte. Es wäre zur Klarstellung dieses anscheinend paradoxen Verhaltens wünschenswert gewesen, Viscositätsbestimmungen des Blutes zu machen, die aus äußeren Gründen leider unterbleiben mußten. U. E. liegt dieser Erscheinung eine Abnahme der Viscosität des Blutes zugrunde.

Nach der Entnahme für die Blutgerinnungsbestimmung erfolgte genau nach dem Bürkerschen Verfahren auch eine Entnahme zur Zählung der Erythrocyten. Die durch Doppelzählungen erhaltenen Resultate zeigen Schwankungen, die bis auf eine einmalige etwas größere Abweichung (Fall V) innerhalb der möglichen Fehlergrenzen liegen, und aus denen sich keine Schlüsse ziehen lassen.

Ebenso ließen die Cholesterinbestimmungen mit Blutproben, die vor Beginn, während und nach Schluß der Faradisation entnommen waren, keine besondere Deutung zu, da auch diese Schwankungen nur ausnahmsweise (Fall V) nicht innerhalb der Fehlergrenzen liegen. Be- 
504 H. Fischer u. E. Thaer: Weiterer Beitrag zur Wirkung der Faradisation usw.

sonders auffallend ist der ungewöhnlich hohe Befund $(0,255)$ in Fall I (genuine Epilepsie).

Der wesentliche Befund unserer Untersuchungen ist der, daß die Blutgerinnungszeit in den Fällen von genuiner Epilepsie und in dem einen Fall von Katatonie mit elementaren Krämpfen (I, II, IV) vor dem Versuch beträchtlich verzögert war, und daß unter der Wirkung der Faradisation sowohl in diesen Fällen wie a uch in Fall III (Myoklonie) und in Fall V (f unktionelle Krämpfe) eine zum Teil ganz erhebliche Verkürzung der Blutgerinnungszeit (bis fast a uf die Hälfte) eintrat. 